\title{
SINR for DS-CDMA with Random Spreading
}

\author{
Ping-Hung Chiang \\ Graduate Institute of Communication \\ Engineering \\ National Taiwan University \\ Taipei, Taiwan, R.O.C. \\ d1942011@ee.ntu.edu.tw
}

\author{
Ding-Bing Lin \\ Department of Electronic Engineering \\ National Taipei University of Technology \\ Taipei, Taiwan, R.O.C. \\ dblin@ntut.edu.tw
}

\author{
Hsueh-Jyh Li \\ Department of Electric Engineering \\ National Taiwan University \\ Taipei, Taiwan, R.O.C. \\ hjli@ew.ee.ntu.edu.tw
}

\begin{abstract}
For wireless multipath fading channels, the performance of a direct-sequence code-division multiple-access (DS-CDMA) system is degraded by the interferences, including the multipath interference (MPI) and the multiuser interference (MUI). In this paper, for the downlink DS-CDMA with random spreading, we derive the variances of the interferences and obtain the signal-to-interference-plus-noise ratio (SINR). The simple and elegant expressions for the variances of the interferences provide useful insights, while the corresponding SINR representation is helpful in evaluating the bit-error-rate (BER) performance that takes the impact of the interferences into consideration. In order to demonstrate our approach, an example regarding the performance analysis of the two-inputsingle-output (2ISO) space-time block coded DS-CDMA with RAKE reception is also provided.
\end{abstract}

Keywords-Antenna diversity, DS-CDMA, MPI, MUI, RAKE reception, random spreading, SINR.

\section{INTRODUCTION}

For wireless multipath fading channels, the performance of a direct-sequence code-division multiple-access (DS-CDMA) system is degraded by the interferences, which are classified into two categories [1]: 1) the multipath interference (MPI), consisting of the intersymbol interference (ISI) and the interpath interference (IPI), due to multipath propagation; 2) the multiuser interference (MUI) from other active users.

Recently, Bjerke et al. analyzed the bit-error-rate (BER) performances of the downlink DS-CDMA with various antenna diversity schemes [2], including both transmit diversity and receive diversity. However, they assumed perfect spreading and hence the impacts of the MPI and the MUI on the system performance are not taken into account.

In this paper, for the downlink DS-CDMA with random spreading, we derive the variances of the interferences and obtain the signal-to-interference-plus-noise ratio (SINR). The simple and elegant expressions for the variances of the interferences can provide useful insights. Moreover, the impacts of the interferences can be included in the derived BER expressions by applying the concept of effective signalto-noise ratio (SNR) to the results in [2]. To demonstrate our strategy, an example regarding the performance analysis of the two-input-single-output (2ISO) space-time block coded DSCDMA (STBC-DS-CDMA) with RAKE reception is also provided.

The rest of this paper is organized as follows. In Section II, the system model and the statistical properties are described.
An example for demonstrating our strategy is given in Section III. Then some numerical results are shown in Section IV, whereas conclusions are drawn in Section V.

\section{PRELIMINARIES}

In this section, the system model of the DS-CDMA is described and some statistical properties including the variances of the interferences are given. To simplify the derivation, we consider the SISO synchronous downlink.

\section{A. System Model}

Let $X_{k, i}$ denote the $i^{\text {th }}$ information symbol of the $k^{\text {th }}$ user, and let $T_{S}$ represent the symbol duration. After short code spreading, the transmitted sequence for all $K$ users in the $i^{\text {th }}$ symbol interval is expressed as

$$
\tilde{s}_{i, l}=\sum_{k=0}^{K-1} X_{k, i} b_{k, l}, \text { for } 0 \leq l \leq L-1,
$$

where $\left\{b_{k, l}, 0 \leq l \leq L-1\right\}$ is the random spreading sequence, taking on the values of $\pm 1 / \sqrt{L}$ with equal probability. Thus, $T_{S}=L T_{C}$, where $L$ and $T_{C}$ represent the spreading factor and the chip duration (i.e. the reciprocal of the system bandwidth), respectively. Assuming that $\tilde{s}_{i, l}$ is zero for $l<0$ and $l \geq L$, the total transmitted baseband sequence is

$$
s_{l}=\sum_{i=-\infty}^{\infty} \tilde{s}_{i, l-i L} .
$$

In this paper, we consider the WSSUS Rayleigh fading channel which can be modeled as a tapped delay line model [3] with fixed tap spacing $T_{C}$. Assuming perfect synchronization and power control, the received baseband sequence of the desired user, denoted by the $d^{\text {th }}$ user, can be expressed as

$$
r_{d, l}=\sum_{m=0}^{M-1} h_{d, m, l} s_{l-m}+n_{d, l}=\sum_{i=-\infty}^{\infty} \sum_{m=0}^{M-1} h_{d, m, l} \tilde{s}_{i, l-m-i L}+n_{d, l},
$$

where $M$ is the number of channel taps, $h_{d, m, l}$ is the tap coefficient with mean zero and variance $\sigma_{d, m}^{2}$, and $n_{d, l}$ is the AWGN with mean zero and variance $N_{0}$. Assume the channels corresponding to different users are identical. Subsequently, the subscript $d$ of $h_{d, m, l}, \sigma_{d, m}^{2}$, and $n_{d, l}$ is omitted for simplicity. As shown in Fig. 1, given $M<L$, rearranging $r_{d, l}$ symbol by symbol as $r_{d, i, l}=r_{d, i L+l}$ yields

This work was supported by the National Science Council of Republic of China under the Grant NSC 92-2219-E-002-010 and the Ministry of Education Program for promoting academic excellence of universities under the Grant 89E-FA06-2-4-7. 


$$
r_{d, i, l}=\left\{\begin{aligned}
\sum_{m=0}^{l} h_{m, i, l} \tilde{s}_{i, l-m}+\sum_{m=l+1}^{M-1} h_{m, i, l} \tilde{s}_{i-1, l-m+L}+n_{i, l}, & \text { if } 0 \leq l \leq M-2 ; \\
\sum_{m=0}^{M-1} h_{m, i, l} \tilde{s}_{i, l-m}+n_{i, l}, & \text { if } M-1 \leq l \leq L-1,
\end{aligned}\right.
$$

with $h_{m, i, l}=h_{m, i L+l}$ and $n_{i, l}=n_{i L+l}$. Inserting (1) into (4) gives

$$
r_{d, i, l}=\left\{\begin{array}{lr}
\sum_{k=0}^{K-1} X_{k, i} \sum_{m=0}^{l} h_{m, i, l} b_{k, l-m}+\sum_{k=0}^{K-1} X_{k, i-1} \sum_{m=l+1}^{M-1} h_{m, i, l} b_{k, l-m+L} \\
+n_{i, l}, \quad & \text { if } 0 \leq l \leq M-2 ; \\
\sum_{k=0}^{K-1} X_{k, i} \sum_{m=0}^{M-1} h_{m, i, l} b_{k, l-m}+n_{i, l}, & \text { if } M-1 \leq l \leq L-1 .
\end{array}\right.
$$

Assume the RAKE receiver has $F$ ( $F \leq M$ ) fingers and, for the finger $f, 0 \leq f \leq F-1$, define (see Fig. 1)

$$
\tilde{r}_{d, f, i, l}= \begin{cases}r_{d, i, l+f}, & \text { if } 0 \leq l \leq L-f-1 ; \\ r_{d, i+1, l+f-L}, & \text { if } L-f \leq l \leq L-1 .\end{cases}
$$

Then substituting (5) into (6) produces

$$
\tilde{r}_{d, f, i, l}=\left\{\begin{array}{l}
\sum_{k=0}^{K-1} X_{k, i} \sum_{m=0}^{l+f} h_{m, i, l+f} b_{k, l+f-m} \\
+\sum_{k=0}^{K-1} X_{k, i-1} \sum_{m=l+f+1}^{M-1} h_{m, i, l+f} b_{k, l+f-m+L}+n_{i, l+f}, \\
\text { if } 0 \leq l \leq M-f-2 ; \\
\sum_{k=0}^{K-1} X_{k, i} \sum_{m=0}^{M-1} h_{m, i, l+f} b_{k, l+f-m}+n_{i, l+f}, \\
\text { if } M-f-1 \leq l \leq L-f-1 ; \\
\sum_{k=0}^{K-1} X_{k, i+1} \sum_{m=0}^{l+f-L} h_{m, i, l+f-L} b_{k, l+f-m-L} \\
+\sum_{k=0}^{K-1} X_{k, i} \sum_{m=l+f-L+1}^{M-1} h_{m, i, l+f-L} b_{k, l+f-m}+n_{i, l+f-L}, \\
\text { if } L-f \leq l \leq L-1 .
\end{array}\right.
$$

Accordingly, at the receiver of the desired user, the codematched filter corresponding to the $f^{\text {th }}$ path performs despreading on $\left\{\tilde{r}_{d, f, i, l}, 0 \leq l \leq L-1\right\}$ and outputs signal for the $i^{\text {th }}$ symbol as

$$
\begin{aligned}
R_{d, f, i} & =\sum_{l=0}^{L-1} \tilde{r}_{d, f, i, l} b_{d, l} \\
& =H_{f, i} X_{d, i}+P_{d, f, i}+S_{d, f, i}+U_{d, f, i}+N_{f, i},
\end{aligned}
$$

where the multiplicative distortion (MD) $H_{f, i}$, the IPI $P_{d, f, i}$, the ISI $S_{d, f, i}$, the MUI $U_{d, f, i}$, and the noise $N_{f, i}$ are given by

$$
H_{f, i}=\frac{1}{L} \sum_{l=0}^{L-1} h_{f, i, l},
$$

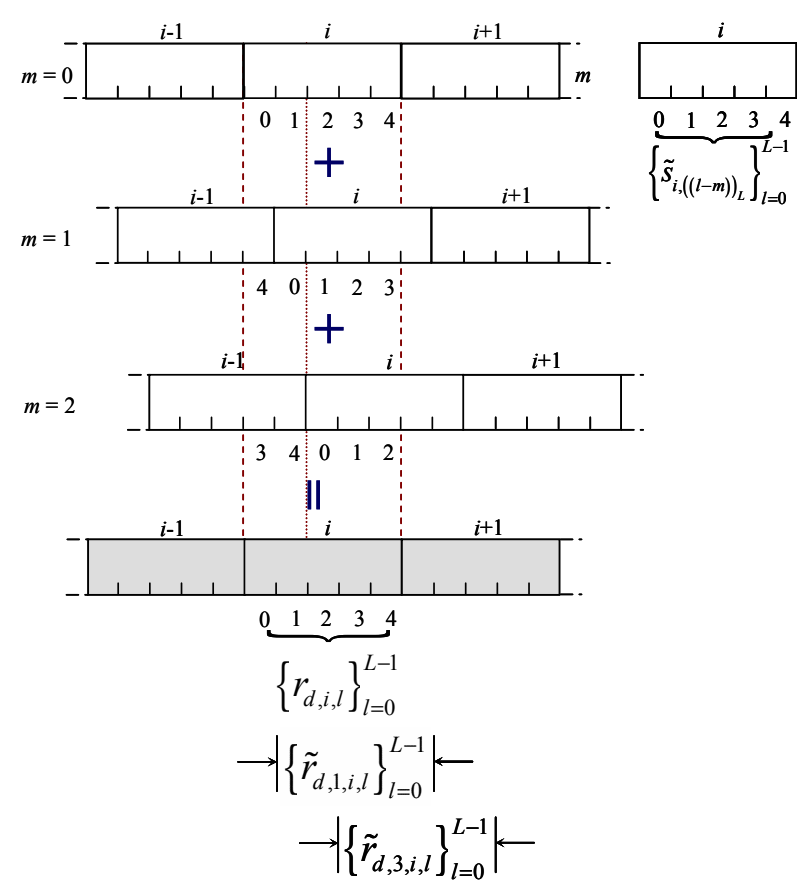

Fig. 1 Graphical illustration of the received signals for $M=3, L=5$.

$$
\begin{aligned}
& P_{d, f, i}=X_{d, i}\left(\sum_{\substack{m=0 \\
m \neq f}}^{M-1} \sum_{l=m}^{L-1} h_{m, i, l} b_{d, l-m} b_{d, l-f}\right. \\
& +\sum_{\substack{m=1 \\
m \neq f}}^{M-1} \sum_{l=0}^{m-1} h_{m, i, l} b_{d, l-m+L} b_{d, l-f+L}-\sum_{m=0}^{f-1} \sum_{l=m}^{f-1} h_{m, i, l} b_{d, l-m} b_{d, l-f+L} \\
& \left.-\sum_{m=f+1}^{M-1} \sum_{l=f}^{m-1} h_{m, i, l} b_{d, l-m+L} b_{d, l-f}\right), \\
& S_{d, f, i}=X_{d, i-1} \sum_{m=f+1}^{M-1} \sum_{l=f}^{m-1} h_{m, i, l} b_{d, l-m+L} b_{d, l-f} \\
& +X_{d, i+1} \sum_{m=0}^{f-1} \sum_{l=m}^{f-1} h_{m, i, l} b_{d, l-m} b_{d, l-f+L}, \\
& U_{d, f, i}=\sum_{\substack{k=0 \\
k \neq d}}^{K-1}\left[X _ { k , i } \left(\sum_{m=0}^{M-1} \sum_{l=m}^{L-1} h_{m, i, l} b_{k, l-m} b_{d, l-f}\right.\right. \\
& +\sum_{m=1}^{M-1} \sum_{l=0}^{m-1} h_{m, i, l} b_{k, l-m+L} b_{d, l-f+L}-\sum_{m=0}^{f-1} \sum_{l=m}^{f-1} h_{m, i, l} b_{k, l-m} b_{d, l-f+L} \\
& \left.-\sum_{m=f+1}^{M-1} \sum_{l=f}^{m-1} h_{m, i, l} b_{k, l-m+L} b_{d, l-f}\right) \\
& +X_{k, i-1} \sum_{m=f+1}^{M-1} \sum_{l=f}^{m-1} h_{m, i, l} b_{k, l-m+L} b_{d, l-f} \\
& \left.+X_{k, i+1} \sum_{m=0}^{f-1} \sum_{l=m}^{f-1} h_{m, i, l} b_{k, l-m} b_{d, l-f+L}\right],
\end{aligned}
$$




$$
N_{f, i}=\sum_{l=0}^{L-1} n_{i, l} b_{d,((l-f))_{L}} .
$$

Notice that (9)-(13) are derived by changing variables, switching the order of the summations, and rearranging the resultant terms. Here $((\cdot))_{L}$ denotes the modulo- $L$ operation, and the subscript $d$ of $H_{d, f, i}$ and $N_{d, f, i}$ is omitted.

From (13), for random spreading, it is readily to recognize that $N_{f, i}$ is still the AWGN with mean zero and variance $N_{0}$. It is assumed that the symbols $X_{k, i}$ are independently and identically distributed (i.i.d.) with mean zero and variance $E_{S}$ (symbol energy) and the tap coefficients $\left\{h_{m, i, l}, 0 \leq m \leq M-1\right\}$ are mutually uncorrelated. Thereupon, even though the distributions of the interferences, including $P_{d, f, i}, S_{d, f, i}$, and $U_{d, f, i}$ are not easy to identify, it is readily to verify: 1) they are mutually uncorrelated; 2) they are all uncorrelated to the MD $H_{f, i}$ and the noise $N_{f, i}$, as well; 3) they are all of mean zero. Furthermore, since independent Gaussian noise results in the smallest capacity, it is reasonable to model these interferences as Gaussian random variables and hence to achieve the performance bound [4]. Indeed, (8) can be rewritten as

$$
R_{d, f, i}=H_{f, i} X_{d, i}+W_{d, f, i},
$$

where $W_{f, i}=P_{d, f, i}+S_{d, f, i}+U_{d, f, i}+N_{f, i}$ is the equivalent AWGN with mean zero and variance $\sigma_{W_{d, f}}^{2}=\sigma_{P_{d, f}}^{2}+\sigma_{S_{d, f}}^{2}$ $+\sigma_{U_{d, f}}^{2}+N_{0}$.

\section{B. Statistical Properties}

Here the statistical properties regarding the channel tap coefficient, the MD, and the interferences are clarified.

For WSSUS Rayleigh fading channels with classical Doppler spectrum, the correlation of the tap coefficients is [3]

$$
\mathrm{E}\left[h_{m, i, l} h_{m^{\prime}, i^{\prime}, l^{\prime}}^{*}\right]=\sigma_{m}^{2} J_{0}\left\{2 \pi f_{D} T_{C}\left[\left(i-i^{\prime}\right) L+\left(l-l^{\prime}\right)\right]\right\} \delta_{m m^{\prime}},
$$

where $\sigma_{m}^{2}$ is the fading power of the $m^{\text {th }}$ tap, $J_{0}(\cdot)$ is the zeroorder Bessel function of the first kind, $f_{D}$ is the maximum Doppler frequency, and $\delta_{i j}$ is the Kronecker delta. For the exponential power delay profile (PDP) [3] with the constraint $\sum_{m=0}^{M-1} \sigma_{m}^{2}=1$, we have $\sigma_{m}^{2}=\lambda^{m}(1-\lambda) /\left(1-\lambda^{M}\right)$ where $\lambda=e^{-1 / \mathcal{D}}$. The delay control $\mathcal{D}$ dominates the normalized root-mean-square (RMS) delay spread $\tau_{r m s} / T_{C}$.

\section{1) Variance of the Multiplicative Distortion}

In light of (9), it is clear that the MD $H_{f, i}$ is of mean zero and hence variance

$$
\begin{aligned}
\sigma_{H_{f}}^{2} & =\mathrm{E}\left[\left|H_{f, i}\right|^{2}\right] \\
& =\frac{\sigma_{f}^{2}}{L^{2}} \sum_{l=-L+1}^{L-1}(L-|l|) J_{0}\left(2 \pi f_{D} T_{C} l\right) .
\end{aligned}
$$

\section{2) Variances of the Interferences}

From (10) and (11), utilizing the fact that the information symbols, the channel tap gains, and the random spreading sequences are mutually independent, the variances of the IPI $P_{d, f, i}$ and the ISI $S_{d, f, i}$ are calculated as follows.

$$
\begin{aligned}
& \sigma_{P_{d, f}}^{2}=\mathrm{E}\left[\left|P_{d, f, i}\right|^{2}\right]=\frac{E_{S}}{L^{2}}\left\{\sum_{\substack{m=0 \\
m \neq f}}^{M-1}[(L-m)+m] \sigma_{m}^{2}\right. \\
&\left.-\sum_{m=f+1}^{M-1}(m-f) \sigma_{m}^{2}-\sum_{m=0}^{f-1}(f-m) \sigma_{m}^{2}\right\} \\
&= \frac{E_{S}}{L^{2}} \sum_{\substack{m=0 \\
m \neq f}}^{M-1}(L-|m-f|) \sigma_{m}^{2}, \\
& \sigma_{S_{d, f}}^{2}=\mathrm{E}\left[\left|S_{d, f, i}\right|^{2}\right]=\frac{E_{S}}{L^{2}} \sum_{\substack{m=0 \\
m \neq f}}^{M-1}|m-f| \sigma_{m}^{2} .
\end{aligned}
$$

Thereupon, the variance of the MPI is

$$
\sigma_{P_{d, f}}^{2}+\sigma_{S_{d, f}}^{2}=\frac{E_{S}}{L} \sum_{\substack{m=0 \\ m \neq f}}^{M-1} \sigma_{m}^{2}=\frac{E_{S}}{L}\left(1-\sigma_{f}^{2}\right) \leq \frac{E_{S}}{L} .
$$

Similarly, by (12), the variance of the MUI $U_{d, f, i}$ is derived as

$$
\begin{aligned}
\sigma_{U_{d, f}}^{2} & =\mathrm{E}\left[\left|U_{d, f, i}\right|^{2}\right]=\frac{E_{S}}{L^{2}} \sum_{\substack{k=0 \\
k \neq d}}^{K-1} \sum_{m=0}^{M-1}(L-|m-f|+|m-f|) \sigma_{m}^{2} \\
& =\frac{E_{S}}{L}(K-1) .
\end{aligned}
$$

Consequently, the power of the total interference can be expressed by a simple formula as

$$
\sigma_{P_{d, f}}^{2}+\sigma_{S_{d, f}}^{2}+\sigma_{U_{d, f}}^{2}=\frac{E_{S}}{L}\left[\left(1-\sigma_{f}^{2}\right)+(K-1)\right] .
$$

Noteworthily, an upper bound of (21) can be obtained by considering the most dispersive condition of the exponential PDP. In this extreme case, $\mathcal{D} \rightarrow \infty, \lambda \rightarrow 1$, and $\sigma_{f}^{2} \rightarrow 1 / M$, i.e., the exponential PDP reduces to the uniform PDP. Hence, for this extreme channel condition, the power of the total interference is given by

$$
\begin{aligned}
\sigma_{P_{d, f}}^{2}+\sigma_{S_{d, f}}^{2}+\sigma_{U_{d, f}}^{2} & =\frac{E_{S}}{L}\left[\left(1-\frac{1}{M}\right)+(K-1)\right] \\
& \leq \frac{E_{S}}{L}[1+(K-1)]=\frac{E_{S}}{L} K,
\end{aligned}
$$

which reveals that the MPI can be treated as the MUI from an additional user. In other words, the total interference can be approximated as the MUI contributed by $K$ users. This confirms the assumption in [1]. Moreover, it is evident that the number of users $K$ dominates the quantity of the total interference whereas the spreading factor $L$ stands for the inherent interference rejection ability of a DS-CDMA system. 


\section{2ISO DS-CDMA}

To demonstrate our strategy, an example for evaluating the performance of the 2ISO space-time block coded DS-CDMA system in the quasi-static (QS) channel is provided. Subsequently, the subscript $d$ is omitted for simplicity.

Denote the spatially identical and independent MD, for the $g^{\text {th }}$ transmit antenna $(g=0,1)$, as $H_{f, i}^{(g)}$ and assume the channel is QS over a space-time codeword duration, then the piecewise-constant MD for the $i^{\text {th }}$ space-time codeword is defined as $\mathcal{H}_{f, i}^{(g)} \triangleq H_{f, 2 i+0}^{(g)}=H_{f, 2 i+1}^{(g)}$ with mean zero and variance $\sigma_{f}^{2}$. Accordingly, from (14), the output for the $f^{\text {th }}$ finger is [2] [5]

$$
\begin{aligned}
& \mathbf{r}_{f, i}=\mathbf{H}_{f, i} \mathbf{x}_{i}+\mathbf{w}_{f, i} \\
& {\left[\begin{array}{l}
R_{f, 2 i+0} \\
R_{f, 2 i+1}^{*}
\end{array}\right]=\left[\begin{array}{cc}
\mathcal{H}_{f, i}^{(0)} & \mathcal{H}_{f, i}^{(1)} \\
\mathcal{H}_{f, i}^{(1)^{*}} & -\mathcal{H}_{f, i}^{(0) *}
\end{array}\right]\left[\begin{array}{l}
X_{2 i+0} \\
X_{2 i+1}
\end{array}\right]+\left[\begin{array}{c}
W_{f, 2 i+0} \\
W_{f, 2 i+1}^{*}
\end{array}\right],}
\end{aligned}
$$

where $\mathbf{w}_{f, i} \sim \mathcal{C N}\left(\mathbf{0}, \sigma_{W_{f}}^{2} \mathbf{I}_{2}\right)$ and $\sigma_{W_{f}}^{2}=2\left(\sigma_{P_{f}}^{2}+\sigma_{S_{f}}^{2}+\sigma_{U_{f}}^{2}+N_{0}\right)$.

Performing the space-time matched filtering gives

$$
\begin{aligned}
\widehat{\mathbf{r}}_{f, i} & =\left(\boldsymbol{\Lambda}_{f, i} \mathbf{H}_{f, i}\right)^{H} \mathbf{r}_{f, i} \\
& =\left(\boldsymbol{\Lambda}_{f, i} \mathbf{H}_{f, i}^{H} \mathbf{H}_{f, i}\right) \mathbf{x}_{i}+\left(\boldsymbol{\Lambda}_{f, i} \mathbf{H}_{f, i}^{H}\right) \mathbf{w}_{f, i} \\
& =\widehat{\mathbf{H}}_{f, i} \mathbf{x}_{i}+\widehat{\mathbf{w}}_{f, i},
\end{aligned}
$$

where $\boldsymbol{\Lambda}_{f, i}=\alpha_{f, i}^{-1} \mathbf{I}_{2}, \quad \hat{\mathbf{H}}_{f, i}=\alpha_{f, i} \mathbf{I}_{2}, \quad \alpha_{f, i}=\sqrt{\sum_{g=0}^{1}\left|\mathcal{H}_{f, i}^{(g)}\right|^{2}}$, $\widehat{\mathbf{r}}_{f, i}=\left[\begin{array}{ll}\widehat{R}_{f, 2 i+0} & \widehat{R}_{f, 2 i+1}\end{array}\right]^{T}, \quad \widehat{\mathbf{w}}_{f, i}=\left[\begin{array}{ll}\widehat{W}_{f, 2 i+0} & \widehat{W}_{f, 2 i+1}\end{array}\right]^{T} \quad$ and $\widehat{\mathbf{w}}_{f, i} \sim \mathcal{C N}\left(\mathbf{0}, \sigma_{W_{f}}^{2} \mathbf{I}_{2}\right)$.

Thereupon, (24) can be separated into two equations as

$$
\widehat{R}_{f, 2 i+t}=\alpha_{f, i} X_{2 i+t}+\widehat{W}_{f, 2 i+t}, \quad \text { for } t=0,1 .
$$

\section{A. Maximum-Likelihood Detection}

From (25), stacking up $\left\{\widehat{R}_{f, 2 i+t}, 0 \leq f \leq F-1\right\}$ gives

$$
\widehat{\mathbf{r}}_{2 i+t}=\boldsymbol{\alpha}_{i} X_{2 i+t}+\widehat{\mathbf{w}}_{2 i+t}, \quad \text { for } t=0,1,
$$

where $\widehat{\mathbf{r}}_{2 i+t}=\left[\begin{array}{llll}\hat{R}_{0,2 i+t} & \widehat{R}_{1,2 i+t} & \cdots & \widehat{R}_{F-1,2 i+t}\end{array}\right]^{T}, \boldsymbol{\alpha}_{i}=\left[\begin{array}{ll}\alpha_{0, i} & \alpha_{1, i}\end{array}\right.$ $\left.\cdots \quad \alpha_{F-1, i}\right]^{T}$ and $\quad \widehat{\mathbf{w}}_{2 i+t}=\left[\begin{array}{llll}\hat{W}_{0,2 i+t} & \widehat{W}_{1,2 i+t} & \cdots & \widehat{W}_{F-1,2 i+t}\end{array}\right]^{T}$. Since $\widehat{\mathbf{w}}_{2 i+t} \sim \mathcal{C N}(\mathbf{0}, \boldsymbol{\Sigma} \boldsymbol{\Sigma})$ and $\boldsymbol{\Sigma}=\operatorname{diag}\left\{\sigma_{W_{0}}, \sigma_{W_{1}}, \cdots, \sigma_{W_{F-1}}\right\}$, then the maximum-likelihood (ML) detection is given by

$$
\begin{aligned}
\hat{X}_{2 i+t} & =\underset{X}{\arg \min }\left(\widehat{\mathbf{r}}_{2 i+t}-\boldsymbol{\alpha}_{i} X\right)^{H}(\boldsymbol{\Sigma} \boldsymbol{\Sigma})^{-1}\left(\widehat{\mathbf{r}}_{2 i+t}-\boldsymbol{\alpha}_{i} X\right) \\
& =\underset{X}{\arg \min }\left\|\boldsymbol{\Sigma}^{-1}\left(\widehat{\mathbf{r}}_{2 i+t}-\boldsymbol{\alpha}_{i} X\right)\right\|^{2}, \text { for } t=0,1,
\end{aligned}
$$

where $\Sigma^{-1}$ represents the noise balancing. Let

$$
\widehat{\mathbf{r}}_{2 i+t}=\boldsymbol{\Sigma}^{-1} \widehat{\mathbf{r}}_{2 i+t}=\boldsymbol{\alpha}_{i} X_{2 i+t}+\widehat{\mathbf{w}}_{2 i+t}, \quad \text { for } t=0,1,
$$

with $\boldsymbol{\alpha}_{i}=\Sigma^{-1} \boldsymbol{\alpha}_{i}, \widehat{\mathbf{w}}_{2 i+t}=\boldsymbol{\Sigma}^{-1} \widehat{\mathbf{w}}_{2 i+t}$. Then, (27) is rewritten as

$$
\hat{X}_{2 i+t}=\underset{X}{\arg \min }\left\|\hat{\mathbf{r}}_{2 i+t}-\boldsymbol{\alpha}_{i} X\right\|^{2}=\underset{X}{\arg \min }\left|Y_{2 i+t}-X\right|^{2},
$$

where

$$
Y_{2 i+t}=\frac{\boldsymbol{\alpha}_{i}^{T} \widehat{\mathbf{r}}_{2 i+t}}{\left\|\boldsymbol{\alpha}_{i}\right\|^{2}}=X_{2 i+t}+Z_{2 i+t},
$$

and $Z_{2 i+t}=\underline{\boldsymbol{\alpha}}_{i}^{T} \widehat{\mathbf{w}}_{2 i+t} /\left\|\boldsymbol{\alpha}_{i}\right\|^{2}$. From (29), it is clear that the $2 F^{\text {th }}$ order diversity channel is transformed into the equivalent AWGN channel via the maximum-ratio combining (MRC).

\section{B. SINR and BER}

From (30), the total instantaneous SINR is derived as

$$
\begin{aligned}
\gamma & =\frac{E\left[\left|X_{2 i+t}\right|^{2}\right]}{E\left[\left|Z_{2 i+t}\right|^{2}\right]}=\left\|\boldsymbol{\alpha}_{i}\right\|^{2} E_{s}=\left\|\boldsymbol{\Sigma}^{-1} \boldsymbol{\alpha}_{i}\right\|^{2} E_{s} \\
& =\sum_{f=0}^{F-1} \frac{\alpha_{f, i}^{2} E_{s}}{\sigma_{W_{f}}^{2}}=\sum_{g=0}^{1} \sum_{f=0}^{F-1} \frac{\left|\mathcal{H}_{f, i}^{(g)}\right|^{2} E_{s}}{\sigma_{W_{f}}^{2}}=\sum_{g=0}^{1} \sum_{f=0}^{F-1} r_{f}^{(g)},
\end{aligned}
$$

where $r_{f}^{(g)}$ is the instantaneous SINR of the $(g, f)^{\text {th }}$ branch. Also, $\gamma$ is of probability density function (pdf) as [2, eq.(18)]

$$
p_{r}(\xi)=\sum_{f=0}^{F-1}\left(\frac{B_{f 1}}{\Gamma_{f}}+\frac{B_{f 2}}{\Gamma_{f}^{2}} \xi\right) e^{-\frac{\xi}{\Gamma_{f}}}, \xi \geq 0,
$$

where $\quad B_{f 1}=2 A_{f}^{2} \sum_{\substack{m=0 \\ m \neq f}}^{F-1} \Gamma_{m} /\left(\Gamma_{m}-\Gamma_{f}\right), \quad B_{f 2}=A_{f}^{2}, A_{f}=$ $\prod_{\substack{m=0 \\ m \neq f}}^{F-1} \Gamma_{f} /\left(\Gamma_{f}-\Gamma_{m}\right)$ and $\Gamma_{f}=\mathrm{E}\left[\gamma_{f}^{(g)}\right]$.

From (29), for BPSK modulation, $\hat{X}_{2 i+t}$ is of conditional BER $Q(\sqrt{2 \gamma})$ and hence its average BER is as [2, eq.(19)]

$$
\begin{aligned}
P_{b} & =\int_{0}^{\infty} p_{r}(\xi) Q(\sqrt{2 \xi}) d \xi \\
& =\sum_{f=0}^{F-1}\left[\frac{B_{f 1}}{2}\left(1-\mu_{f}\right)+\frac{B_{f 2}}{4}\left(2-3 \mu_{f}+\mu_{f}^{3}\right)\right],
\end{aligned}
$$

where $\mu_{f}=\sqrt{\Gamma_{f} /\left(1+\Gamma_{f}\right)}$.

\section{NUMERICAL RESULTS}

For all numerical results, the parameters are detailed as follows. Firstly, the system bandwidth is $800 \mathrm{KHz}$, thus the chip duration is $T_{C}=1.25 \mu$ seconds; secondly, for exponential PDP, the number of uncorrelated paths and the delay control are $M=6$ and $\mathcal{D}=2$, respectively, which correspond to the RMS delay spread $\tau_{r m s}=1.74 \mu$ seconds; thirdly, the channel is QS; fourthly, the modulation is BPSK; finally, the number 
of fingers is $F=1,2, \ldots, 6$. In addition to the BERs of the 2ISO DS-CDMA system, the BERs of the SISO system are also provided as the benchmarks.

Fig. 2 is the BERs of various DS-CDMA systems with $K=$ 10 and $L=127$. It is evident that the interferences, comprising the MPI and the MUI, have great impacts on the system performances such that the irreducible error floors occur. In addition, it is clear that the 2ISO systems benefit from the larger diversity gain and hence perform better than the SISO systems. Thereupon, combining the path diversity and the antenna diversity does enhance the signal component and provide the better interference reduction.

Fig. 3 is the error floors corresponding to different spreading factors (i.e. $L=15,31,63,127,255,511,1023$, and 2047), provided that $K=10$. It confirms that enlarging the spreading factor results in a better interference rejection ability of the DS-CDMA system and hence a lower BER value.

Fig. 4 is the error floors with respect to different number of active users, given that $L=127$. For $F=6$, if the permissible BER is $10^{-3}$, then the maximum numbers of active users for the SISO and the 2ISO systems are about 12 and 18, respectively. Consequently, by adding one transmit antenna at the base station, the system capacity is increased about 6 users.

\section{CONCLUSION}

In this paper, for the downlink DS-CDMA with random spreading, we derive the variances of the interferences and obtain the SINR. The simple and elegant expressions for the variances of the interferences provide useful insights, while the resulting SINR representation is helpful in evaluating the BER performance. An example regarding the performance analysis of the 2ISO space-time block coded DS-CDMA with RAKE reception is also provided. Based on our approach, by applying the concept of effective SNR to the results in [2], one can theoretically investigate the impacts of the MPI and the MUI on the performance of the DS-CDMA systems.

\section{REFERENCES}

[1] T. Eng and L. Milstein, "Coherent DS-CDMA performance in Nakagami multipath fading," IEEE Trans. Commun., vol. 43, pp. 11341143, Feb./Mar./Apr. 1995.

[2] B.A. Bjerke, Z. Zvonar, and J. G. Proakis, "Antenna diversity combining schemes for WCDMA systems in fading multipath channels," IEEE Trans. Wireless Commun., vol. 3, pp. 97-106, Jan. 2004.

[3] G. L. Stüber, Principles of Mobile Communication, $2^{\text {nd }}$ ed. London: Kluwer Academic Publishers, 2001.

[4] C. E. Shannon, "Communication in the presence of noise," Proc. IRE, Vol. 37, pp. 10-21, 1949.

[5] S. M. Alamouti, "A simple transmit diversity scheme for wireless communications," IEEE J. Select. Areas Commun., vol. 16, pp. 14511458, Oct. 1998.

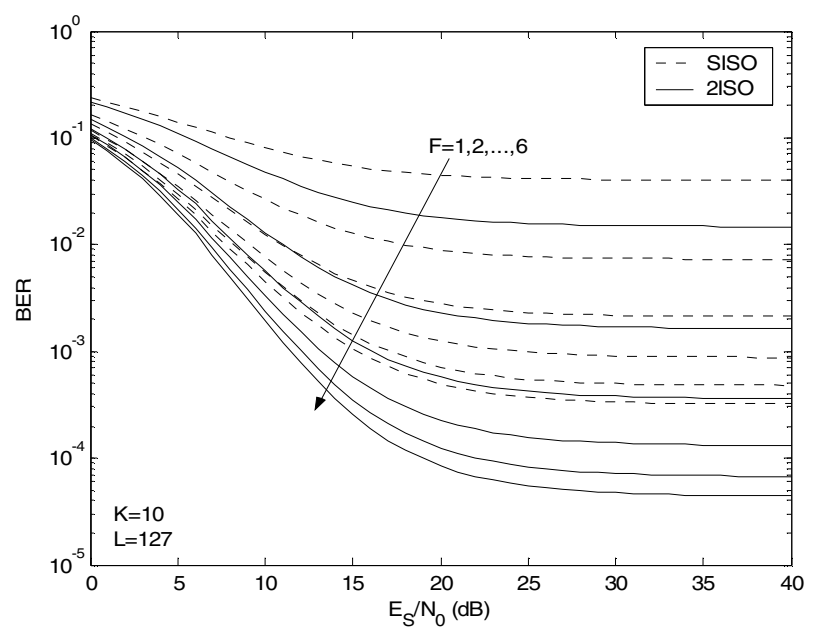

Fig. 2 BER versus SNR.

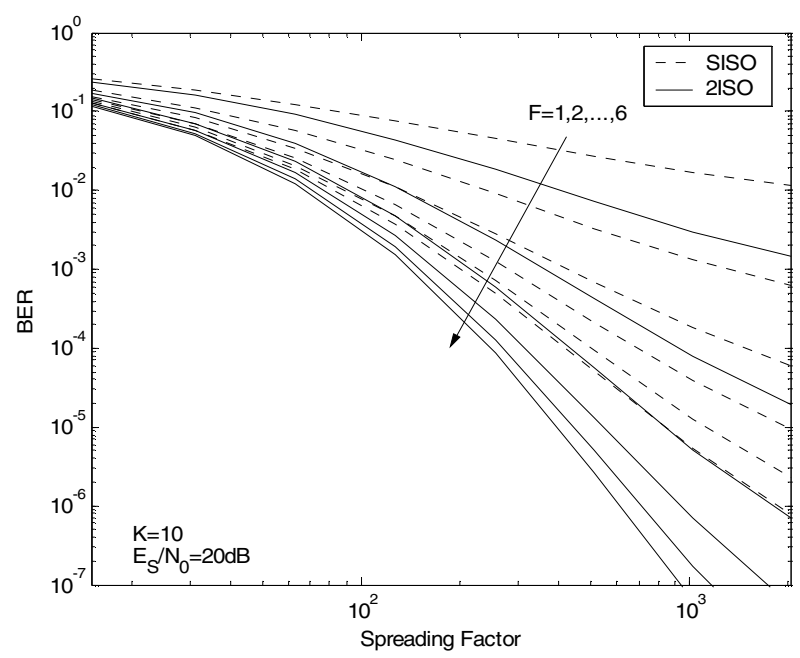

Fig. 3 BER versus $L$.

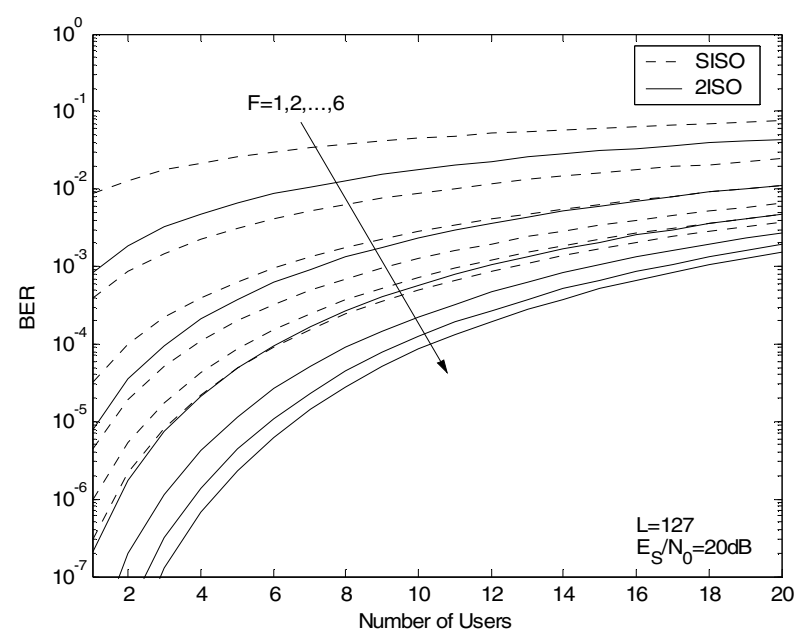

Fig. 4 BER versus $K$. 